\title{
Using ANP to design a living system like balanced operating model for intangible services
}

\author{
Angela Minzoni * \\ Ecole Centrale Paris / Laboratoire Génie industriel \\ Chatenay-Malabry \\ France \\ Chair. Operational Efficiency and Management Systems \\ Corporate chair BNP Paribas / ECP \\ angela.minzoni@ecp.fr \\ Eléonore Mounoud * \\ Ecole Centrale Paris / Laboratoire Génie industriel \\ Chatenay-Malabry \\ France \\ Chair. Operational Efficiency and Management Systems \\ Corporate chair BNP Paribas / ECP \\ eleonore.mounoud@ecp.fr \\ Majid FathiZahraei \\ Post doctoral Fellow. Operational Efficiency and Management Systems \\ Corporate chair BNP Paribas / ECP \\ Ecole Centrale Paris / Laboratoire Génie industriel \\ Chatenay-Malabry \\ France \\ majid.zahraei@ecp.fr \\ the authors are thankful to Jean Guy Caputo and Antonmaria Minzoni Alessio for their \\ contribution in the understanding of mathematical concepts applied to the study of living
} and dynamic systems

\begin{abstract}
ANP and AHP based approach for weighting criteria within decision making contexts has no need to be presented once more. Academic literature in disciplines like economics, engineering, political sciences, statistics or mathematics testify of the broadness of topics, situations and cultures where the method' s value has been proven. From supply planning or road mapping to monitoring, from crisis management or banking crime to rural water supply, decision makers - both in governments or firms- have implemented this method in the five continents.
\end{abstract}

Less has been done in the use of the method beyond decision making, as for example in the field of innovation or organisational design where it can be expected that ANP-AHP facilitates the formulation of a suitable dynamics to inter-wave the complex links of 
experts' representations of the system under study. We advance the hypothesis that the rough data friendliness of ANP-AHP method, combining tangible and intangible information can be used to provide the relevant interactions for living systems like modelling which is at the centre of the cross disciplinary creativity needed to design innovative and balanced operating models

Keywords : innovation, design, strategy, operations, intangible services

\section{1) Introduction}

This study was motivated by one basic assumption and two main issues stemming from academic literature and organization' s observation. The basic assumption states that operating models for the tangible, manufacturing industry are less and less adequate for intangible knowledge and information based services. Excessive process optimisation has lessen the global vision of the system in which processes take place. There is a need to find new ways of linking the global and in particular, understanding their dynamic interactions among the macro and the micro levels other than the well-known traditional subordinate alignment.

Operating model in this study is understood under a broad perspective focusing not only on the way processes are engineered and operated but also including strategy, governance and resources issues. This we have named "operating model from the governance to the tools". This basic assumption underlines the search for a new operating idem model design focused on balance, this being understood through the metaphor of the living systems long lasting homeostatic regulations.

The two main issues are the following:

- the elaboration of a cross-disciplinary integrated research methodology (including organizational sciences, anthropology and operations research), able to help the organization to design an innovative operating model thus contributing to academic research by innovating in terms of methodology.

- the use of Analytic Network Process (ANP) method for a design purpose, that is, beyond the traditional decision making context. This new kind of application leads to examine in depth the organizational features at stake in short or medium term decision making contexts and long term prospective design contexts. This approach also leads to examine the role of experts in these two different contexts even if this study focuses in organizational design. We postulate that ANP approach can be central to such a methodology as it is able to cope with very diverse parameters, including multiple sloppy factors and to challenge data gathering, expert' $s$ status and legitimacy as well as the role and understanding of subjectivity. We can notice here that ANP literature has not developed until now an in-depth approach on human factors related to experts, to the numeral expression of ratings during pairwise comparisons nor to the expert' $\mathrm{s}$ learning dynamics of their own system as much as it has developed the algorithmic reflection. 
In the following pages we will present in detail a cross-disciplinary action-research initiative in progress. This work is undertaken in a highly knowledge-intensive ITsupported industry. It is involving middle and top managers within the task of designing a new overarching organisational system, encompassing both operational and strategic issues. ANP approach and method, crossed with organization sciences and anthropology one's are used first to assess the criteria involved in the current model and second to design a new model. A key challenge is to be able to go beyond a mere structural description of an operating model in terms of units and linkages.

Operating models are detailed descriptions of how organizations operate across processes, people and technology in order to accomplish their functions. Operating model design should not be confused with detailed design of processes, systems and organization structures. For example, an operating model might specify which business processes are needed - and whether those processes should be outsourced, centralized as shared services or handled by business units and where they should be 'shored' - but would not define the details of the processes to be executed. In particular, operating models indicate the level of process automation and standardization. Current practices such as acumulating processes for standardized products as a way to achieve efficiency has led to high process redundancies and much complicatedness in operating models, often referred to as "bad complexity". Thus a new call for simplicity and simplification is currently made. We believe that a more radically new approach to operating models is needed. Our way of doing this is to look at the field of design, and borrow proven practices of co-creation to foster innovative operating models based upon multidisciplinary insights in the concept of equilibrium.

\section{2) Literature Review}

References have been chosen according to their potential to inspire innovation in the field of operating models under the broad focus "from the governance to the tools". This requires to explore literature - under a cross disciplinary focus- on topics such as governance, strategy, business models, operating models, resilience, design, neural networks, human factor, narratology, mapping, creativity, cybernetics, cognitive sciences, systemic approaches, subjectivity, etc $\cdots$ A special focus has thus been given to literature highlighting complexity, networks and links. This vast topic overview is indispensable to carry out an accurate criteria identification for the multi-criteria modelling of operations as well as to animate the working meetings with experts, who are, at the same time, empowered by the organization to be the members of the design team for the future operating model prototype.

Existing OR literature about multi-criteria issues in general and about operating models in particular is almost exclusively focused on decision making models, According to $\mathrm{Da}$ Silva et al. (2009), before 2003 these methods - and in particular those concerning supply chain issues- used almost exclusively quantitative criteria (for ex : linear weighting models, Total Cost Ownership, Principal Component Analysis). After 2003 more attention began to be given to qualitative criteria and, consequently, actual methods tend to use both criteria. The ISAHP 2014 thus innovates while suggesting to use ANP and

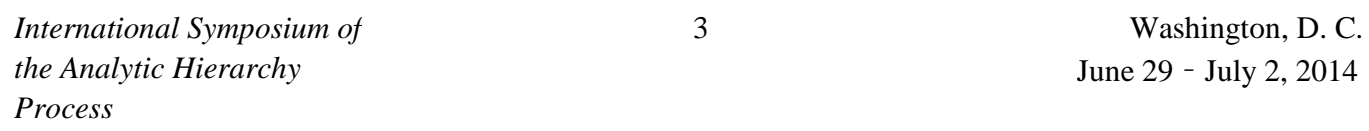


AHP methods for exploring situations beyond decision making. Organizational sciences as well as anthropology will look at operating model multi-criteria issues under a qualitative approach, these disciplines will also be attentive to the ways quantitative data are gathered and given sense. Actually, these disciplines are developed in specific and often separate research departments and institutions. Consequently the present researchin-progress innovates because of the simultaneous integration of three disciplines in a single research department and methodological approach.

We detail the different issues on which these three disciplines focus when they deal with the differences in the ways organisations operate and the consequences it has on their equilibrium. Organizational Research focuses on issues like resilience and management of high-reliability organisations. Operational Research focuses on issues like costs, benefits, opportunities and risks of operating models' characteristics such as outsourcing or offshoring. Anthropology will focus on cross-disciplinary methods, group dynamics, creativity and cultural and cognitive issues. Going beyond decision making needs to develop an integrated research methodology where the issues mentioned above are put at use simultaneously to address N. Bellomo' s (2003) fundamental statement: « the main problem consists in understanding how the qualitative interpretation of social reality, delivered by research in the social sciences, can be transferred into quantitative description produced by mathematical equations ». We can add that this is even more true in domains like governance, strategy or management where data are essentially qualitative and related to notions like power, leadership, followership, influence, intuition, information gaps, experience, trust $\cdots$

Organizational research has been scrutinizing high-reliability organizations (HRO, organizations in which accidents rarely occur despite the error-prone nature of the work such as nuclear plants, aircraft carriers, etc ) for more than 20 years to understand how they are able to operate safely despite challenging operating conditions. As work in many organizations had become increasingly fast-paced and the margin for error has become smaller, the notion of 'high reliability' has received growing attention in many industries. At the core of high reliability organizing is a set of principles embodied in processes and practices that enable organizations to focus attention on emergent problems and to deploy the right set of resources to address those problems. Noticing and responding to small disturbances and vulnerabilities allow people to take corrective action before escalation into crisis, the issue being that small problems are very difficult to detect.

Weick and Suttclife (1999) have characterized HRO by five key principles that facilitate both problem detection and problem management. Problem detection involves three of them: firstly, using failure and near failure as ways to gain insight into the strengths and weaknesses of the system; secondly, avoiding the tendency to minimize or explain away problems; and thirdly being aware of operations interconnectedness and how problems in one area can spread to other areas. Problem management is based upon the last two principles : developing the capability to cope with un-expected events (that is resilience), and understanding where the expertise is in the organization and ensuring that decisions about how to deal with problems are made by those experts (that is expertise recognition or deference). The processes and practices that enact those principles might differ depending on the organization' $\mathrm{s}$ context and the set of resources and constraints that it faces. 
Reliability and safety are difficult to account for: what is not happening (catastrophic error) being more visible than what is happening (timely human adjustments).

Consequently, reliability and safety have been described as 'dynamic non-events' (Weick 1987, 2011): 'dynamic' in the sense that reliability and safety result from managing continuous change and 'non-events' in the sense that we recognize reliability and safety by the absence of errors and accidents). As dynamic non-events, reliability and safety must be recurrently re-accomplished every day. So reliability can be defined as a dynamic set of properties, activities, and responses and thus open the question of the balance or inner equilibrium of the system.

Although the high-reliability organization (HRO) literature identifies several attributes that differentiate HROs from other types of organizations, these studies do not explain how an HRO comes into being with a few exceptions. O'Neil and Krane (2012) built a 97-year longitudinal case study tracking the emergence and continuation of HRO characteristics in the Federal Aviation Administration' $s$ air traffic control operations to tackle that issue. Their analysis shows that HRO characteristics emerged incrementally over an extremely long period of time, and policy changes preceded organizational changes early in the process of HRO development, but the relationship of policy change to organizational change decreased in later stages. These findings should be related to the most recent work on financial markets by Martinez-Moyano et al. (2014) dedicated to rule development, compliance, and organizational change. This study used causal-loop modeling from system dynamics to articulate the tension between production goals that focus on short-term, certain, salient benefits and required adherence to productionconstraining rules that attempt to mitigate long-term, uncertain, non-salient risks. They describe how organizations attend to rules depending on the nature of the benefits of production compared with those of rule compliance. Their model identifies the operative mechanisms responsible for the development of pressures for production and for rule compliance in organizations, providing an explanation both for problem-prone organizations characterized by erosion of standards and increased violations and for organizations following rules more reliably. These two pieces of research encourage us to articulate both organizational and institutional features in our modeling.

ANP method (Saaty, 1999, 2005, 2008) being a discrete multiple criteria one, characterized by the decomposition of a problem in a network structure, without hierarchical relations among its elements, is a suitable approach within the context of the current study. This method allows the establishment of relations of dependence and feedback among criteria and alternatives. The way the problem -or the situation, or the system- is decomposed is thus of paramount importance, this decomposition will be a specific and iterative one where the problem will be stated and decomposed and re stated and recomposed in a quite parallel movement. The stake in the current research being to take advantage of this method to imagine a new system and not to choose between solution alternatives to a well delimited problem. Organization sciences and anthropology have the adequate tools and methodologies to address this question and to facilitate expert's identification of the suitable criteria needed for the ANP, as well as to put at use ANP results towards the final objective. 
Mathematical sciences applied to biology will help to address the dynamic equilibrium and the stability issues. We stress that the particular focus on biology is key to the undergoing research presented in this paper because of its particular non reciprocal and non linear network specificities, moreover the behavior of the operating system under study may depend on more than just the present state and the number of states might be not a finite one.

A convenient definition of equilibrium for our purposes is taken from mathematical modelling in Biology (J.D. Murray, 2008). It is said that the system is in equilibrium provided that its structure and relations are such that its state does not change in time . Moreover if the system is perturbed and recovers the original state we say that the equilibrium is stable.This notion is appropriated for a Fuzzy Cognitive Map (FCM) formulation of the system.

Literature about FCM has also been useful within the context of the present study. First introduced by political scientist R. Axelrod (1976), these maps are causal maps linking goals, values and trends allowing systemic influence propagation, in particular forward and backward chaining and are well adapted to soft knowledge domains (Kosko, 1986, Stylos \& Groumpos, 1999). According to Aguilar (2005), FCM are the fusion of the advances of the fuzzy logic and cognitive maps theories. This approach offers the advantage of quantified results adapted to the understanding of dynamic systems, especially interesting when the systems involve significant nonlinear feedbacks and hard to come or uncertain numerical data.

Mapping is thus recognized in disciplines like politics, anthropology, economics, management sciences, cognitive, educational or environmental sciences just to mention some of them to explore similarities and differences between stakeholders' understanding of an issue and to improve communication between stakeholders. Mapping integrates different perspectives for improving overall understanding of multi-functional system in the case of this study the broad overview of operating models. This technique identifies and overcomes stakeholders' knowledge limitations and misconceptions associated with a given resource (Jones et al., 2011)

Anthropological discussions on operating models are still very few, these will particularly highlight cultural issues among onshore and offshore dynamics (Eaton, 2011) such as the tools of globalization, the round the clock style, the primary and secondary communication channels, the workplace privacy but will also take into account power outages per day, number of laptops needed for virtual meetings, the number of emails and the sending practices linked to this communication. These studies call attention to the fact that predominant theories about offshore outsourcing focus mainly on cost incentives and short term efficiency gains which can lead to an important percent of failures for offshore outsourcing activities because of the neglecting of people related cultural specificities and the obscuring of the longer term potential risks and costs. These theories would also evaluate offshore outsourcing practices from the only perspective of the firm who is looking to outsource. It can be noticed here that operational research papers would rarely take into account the impact of their optimization calculations on people competencies, lack of control over the processes, irregular quality, power relations or hidden costs not to say country policies or regulations.

International Symposium of 6 
Anthropological perspective will also draw the attention upon the local perception of the global and suggest to develop a perspective from the local to the global analyzing how global aspects are put into local frames and how to find locality within globalization (Korff, 2003).

Anthropologists will also stress upon the offshore outsourcing discussions and jokes in the media, television talk shows, cartoons or movies. These media seem to convey stereotypes about low cost- low value activities and people performing them, the yes men and dependent syndromes, electronic shake hands, etc $\cdots$.

As highlighted by the pioneering Chicago School study by Thomas \& Znaniecki between 1918 and 1920 about the crossed impacts of immigration both in the left country and in the newly reached one, the use of narratives is singularly adequate to the expression of complex interwoven intercultural situations like the one under study in this paper.

Cognitivists have also stressed the important role of stories (not to be confused with anecdotes) in the learning process, particularly when the learning degree needed is as high as is the need for thinking up the cycle of the object to come, in this case a new and broad spectrum operating model design.

Narratology techniques in design provide a way of getting a feel for new terrains, tying objectives together, expressing deep basic assumptions and identifying challenges and risks. They advocate the taking into account of the verbal expression of subjectivity as a powerful mean for users and designers to express and address human needs, in particular at the starting point of a design process. Stories are important cognitive events for they encapsulate into one compact package, information, knowledge, context, and emotion. They provide a context for anchoring understanding and for assessing and integrating new knowledge and are much more memorable than facts and figures. This is particularly true at the starting point of a design process where the primary goal is not to produce a functional specification, but get a high level vision of the system components and their dynamic relations.

\section{3) Objectives}

Outlining operations in highly knowledge-intensive IT-supported intangible industries involves operators, middle and top managers -as well as researchers- within the task of designing a new overarching organisational system, encompassing both operational and strategic issues. ANP approach and tools are tested here first to assess the current model' $\mathrm{s}$ fragmented criteria - mainly cost and performance oriented and second to ideate a new model where the main drivers will be the system' $s$ balance and stability.

A key challenge is to be able to go beyond a mere structural description of the multiple units and linkages of each unit cluster of the organisational model. We develop a methodology that allows us to provide an eco-system like functional description of the dependences and interdependences within the system and their impact on the robustness and resilience of the new system. ANP is central to this methodology as it is able to cope with very diverse parameters, including multiple sloppy factors and to challenge data 
gathering, experts' status and legitimacy as well as the role and understanding of subjectivity.

The methodology design here does not refer to the empirical procedures employed for obtaining or eliciting data, but rather to the conditions that must be satisfied if the data are to be judged evidentially relevant for the acceptance or rejection of an explanation or interpretation.

Companies are continuously working to improve their day-to-day operational processes. However, most of them prefer not to fiddle with their operating model unless they have to. In most cases, companies only decide to change their operating model when facing major events such as a merger, business crisis, regulatory shift or new competitor entrance. Event-driven changes are easier to conduct because they are less questioned and can be conducted quickly, based on top-down directive fast decisions. Communication can then be delivered on a need-to-know basis and limited to people who are directly affected. Unfortunately, beyond the quick wins, too many companies falter in their efforts to redefine their operating models while facing financial pressure this limiting investment in new capabilities.

Existing approaches to the development of operating models are based on traditional, linear problem solving techniques. This type of approach can be very effective when uncertainty and complexity are low and when the new design doesn' t need to be radically different than the current one. However, they can not address the needs of the current highly fluid, uncertain environment, where IT supported services need to radically change their operating models in order to survive. All the traditional levers, such as economies of scale, single platforms and shared services, must be cut back, or at least can't be exploited as before. So, high value services are paradoxically always looking for new ways of reducing costs, while coping with a complex and disintegrated value chain while at the same time aiming at delivering excellent customer service. However, traditional operating model improvements based upon continuous improvements rather than design are unlikely to be enough to deliver the cost base reductions looked for. That's why alternative cost reduction measures, such as straight- through processing, first-time resolution and self-service channels are sought. Straight Through Processing (STP) is a good example of the willingness to minimize human input to make staff savings, which usually represent more than half of intangible services' costs. Thus complex IT architectures are needed to accommodate new operating models with greater demands for data.

Thus cost reduction is only part of the challenge. If design is limited to process improvements, the new operating model will never fully achieve any change of logic or even if it includes new structures, accountability principles and key metrics (such as KPI). In addition, equal importance should be given to the interfaces and behavioral assumptions embedded in governance rules (that is defining by whom and how decisions are supposed to be made) and management principles (that is defining with what resources and nature of autonomy people are supposed to work with). To create alternative operating models calls for new design criteria to be identified. For example, companies may have strategies but they will need to make them more granular for setting design criteria along which building operating models. The same reasoning applies to 
governance rules and management principles that have to be translated into design criteria in order to be put at work with the usual suspects, such as cost reduction levers, to build an over-all view of the balance of the operating model.

\section{4) Research Design/Methodology}

An intensive cross-disciplinary qualitative phase allowed us to identify the relevant criteria to be used for modelling under ANP. This phase was conducted by applying methods such as narratology and mapping, both methods being familiar to anthropologists and organizational scientists and recognized as particularly adapted to complex situations.

The main stakes related to the identification of experts according to their position in the organization, are presented here. Three groups of 3 to 5 managers in three different business units have been empowered by the organization to be the members of the design team for the so called 'prototyping' of alternative operating models. Asking these managers to sit in a room as design experts put them in a very different posture compared to being involved as an expert in decision making. Making decision is quite usual for managers whether they are operational managers in charge of running business units or functional ones involved in organisational choices. Decision making is one of the central activities of management. Good decision making is regarded as an essential skill to become an effective manager and for a successful career. Operation Research (OR) is a discipline explicitly devoted to aiding decision makers. Decision making is supposed to be about identifying and choosing alternatives based on values and preferences. But generating alternatives is not prior for managers compared to choosing the one that has the highest probability of success or effectiveness and best fits with the company objectives.

On the contrary the engineering design process focuses on generating alternatives and involves a number of steps, and parts of the process may need to be repeated many times before the process ends. The engineering design process includes research, conceptualization, feasibility assessment, establishing design requirements, preliminary design, detailed design, production planning and tool design, and finally production. Design is often referred to as design science but the distinction between scientific method and design method was introduced as early as in the sixties. Since Simon popularized the term in his argument for the scientific study of the artificial (as opposed to the natural), design science has come to have the meaning of scientific study of design. Furthermore designing a new operating model requires not only rigor but also creativity and a capacity to step away from the present way of thinking, representing and organising operations.

Thus, being an expert involved in a group during a design process is much more challenging than being involved in a decision-making exercise than can sometimes be based upon a single interview. Firstly it means energy dedicated to the project over a long period that we estimate up to two years minimum. Secondly it means participating in a working group with peers, for the group being able to cover expertise over the different areas, from the governance to the tools. Thirdly as excessive process optimisation has lessen the global vision of the system in which processes take place, it can be lengthy and 
difficult for the group to encapsulate their detailed knowledge of daily operation and decision processes into a coherent model. Facing that difficulty and allowing themselves (and feeling allowed) to spend the time necessary to overcome that collective pitfall is not straightforward. Lastly, once a more global view on the current system is reached, more energy is needed to imagine alternatives with a prospective stance. These two last points can be make experts feel puzzled during some working sessions, as they are mainly involved with the current model and its related ways of thinking about operations in their daily life with the limited exceptions of weekly working sessions.

Recent approaches (Ashkenas, 2012) have pointed out the need to innovate in terms of operational efficiency representations and measures within the organizations, especially for intangible service providers. Over-optimization of parcelled up processes has lessen the global vision of the system in which the processes take place. In fact, these firms have on boarded -almost as is- the same ways of thinking familiar to the mechanistic manufacturing industries. Until now, academic research has focused on this kind of industries and, when dealing with services, it will be the same mechanistic approach that will prevail, in short: an everlasting cost reduction never fully achieved objective, a process atomization where parts are left in a country and parts are send to another, very much in a similar way airplanes are assembled or fruits are grown someplace and stewed at the opposite side of the world or phone calls are dispatched worldwide through optimized linear approaches. We can observe, for example, that academic literature (web of science survey 1995-2014) about the onshore/offshore issues - with a leap up between 2007 and 2010 and mainly originated in the US- will focus principally on criteria such as infrastructure and salary costs. The main scientific reviews addressing this issue publish in the following domains: computer science information systems, management or operations research. These publications will rarely be cross-disciplinary, this fact probably contributing to spread the narrow vision echoed in firms. Key words in these contributions will be aligning, control, flexibility, provider' s selection, capability loss, security, conflict of interest, transportation, shortest path, maximum flow, pure minimum cost flow, etc $\cdots$

Let's notice that the Lean management, once deprived of the original Japanese cultural seeds embedded in the main principles conveyed by this approach, has contributed to the atomization of processes and the complication of their management. Indeed, the Lean approach arose almost 25 years ago within a social and business context where database marketing was coming forth, where gaps between the executives and employees salaries were growing deep, where the competition was based on IT systems, where LBO came out as a financial technique, where leaders were seen as negotiators. Today, the world has changed in its political, financial, managerial and social aspirations, thus the need to renew the operational efficiency fundamentals.

Even if the Operational Research scientific community (two examples among many: Padillo \& Diaby, 1999; Cardoen et al, 2010) has mainly focused on production planning or supply chains in manufacturing industries and much less on the intangible, high speed, knowledge based industries like the banking sector, some ideas are insightful, for example:

- the need to include other important criteria besides the monetary element in the analysis of the operating models alternatives, such as: the importance and 
maturity of technology, the risks at stake, the workforce stability, the complexity level in planning, control and supervise, the quality standards, the complexity of end products, the expected volume fluctuations and uncertain volume estimates. Just to cite some of them

- the operating model issue can be approached from an operational point of view or from a technology management perspective depending on what triggers the decision. When the focus of managerial planning is on Operations the operating model analysis is short time framed, tactical and performed in the context of an efficient allocation of resources among different activities. Conversely, triggers such as new product development yield operating model analysis with a predominance of technologically-oriented issues that require a more strategic approach, thus framing the problem within a high level of managerial planning. .

The transfer of manufacturing approaches to service delivery has led to a loss of view of the entire context of service operations, loss even more important than transactions are intangible, electronic and often automated. According to a recent study (Collinson, 2011) the complexity in the banking sector can be described as an accumulation of procedures in order to improve performance. But this so called "bad complexity" do reach a tipping point where costs outweigh benefits. Not to mention directly offshoring activities, Collison's study points out the negative impact of the geographical diversity on banks. It also points out the harm of internal forms of bad complexity, these being even more harmful than external forms.

Over focalisation on just in time, when allowed time for tasks becomes shorter and shorter, with no margins, reduces the scope of the activity, the processes and the responsibilities thus congesting the system contrary to the initial purpose to smooth the system.

It seems clear today that the banking operating models must be carefully designed before they are implemented in order to take into account the multiple parameters influencing operational efficiency or being influenced by it. Among these parameters can be cited the external environment, such as regulators, clients or standards, the institution' s corporate governance, the business strategy, the processes, the procedures and the tools. These parameters are heterogeneous in nature and not all are measurable ones. This challenges traditional OR methodologies which lie upon detailed flow measures and makes methods like ANP very useful as a discrete multiple criteria method, characterized by the decomposition of a problem in a network structure. The method allows relations of dependence and feedback among criteria and alternatives (Saaty, 2005).

Even if the ANP method has been mostly used in decision making contexts, characterized by an organizational specific dynamic between experts and decision makers, this article makes the hypothesis that ANP can bring light during the design process for inventing an innovative understanding of operational efficiency. On the other hand, ANP can also benefit of a particular methodology common in anthropology which mixes the power of maps in visualising the whole system at a glance and the power of narratology, letting experts express their knowledge in a situated and embodied way. In the case of this study, experts are members of the design team and, as such, they are not only data providers for 
researchers but they do contribute to the data processing and the outlining of the future system.

The contribution of ANP methodology within the actual cross-disciplinary design process is shown in Figure 1. Once the criteria have been identified by experts through organizational prototyping meetings methodologically based on narratology and mapping techniques, we have proceeded to a first pairwise comparison round in order to assess consistency cluster per cluster, but also to allow experts to re-view, when and if needed, the initial criteria and their distribution in the map. On the basis of ANP methodology and Pareto law, some criteria were identified as outstanding - using for that the limiting values and will be considered as the "pillars" of each cluster. Inner and Outer dependency links as well as feedback one' s will be explored on the basis of these "pillars" . In this step the intention is to reach an elementary consistent model. The project is today almost at the end of stage 4 (Figure 1)

\section{5) Data/ Model Analysis}

The new research and academic approaches presented here aim to help banks \& knowledge based services to think their own operational efficiency system in other terms than the mechanical one' $\mathrm{s}$ of car like assembling automated factories. This new way is inspired is by the methods used for the understanding of living eco systems. The living system metaphor is also more consistent with renewed approaches not only for organizational design but also for decision making, governance or collaboration as well encouraging new responses to the need for coordination, control, swarm intelligence and collective wisdom.

This methodology was carefully designed to allow from the beginning an end to end vision of the research project while leaving the necessary place for step by step hazards to be taken into account. The research project design focuses the attention on :

- the coordination of a cross-disciplinary integrated conceptual and methodological approach including anthropology, organization sciences and operations research. These three disciplines contributing to the understanding of operations under a living system focus. 


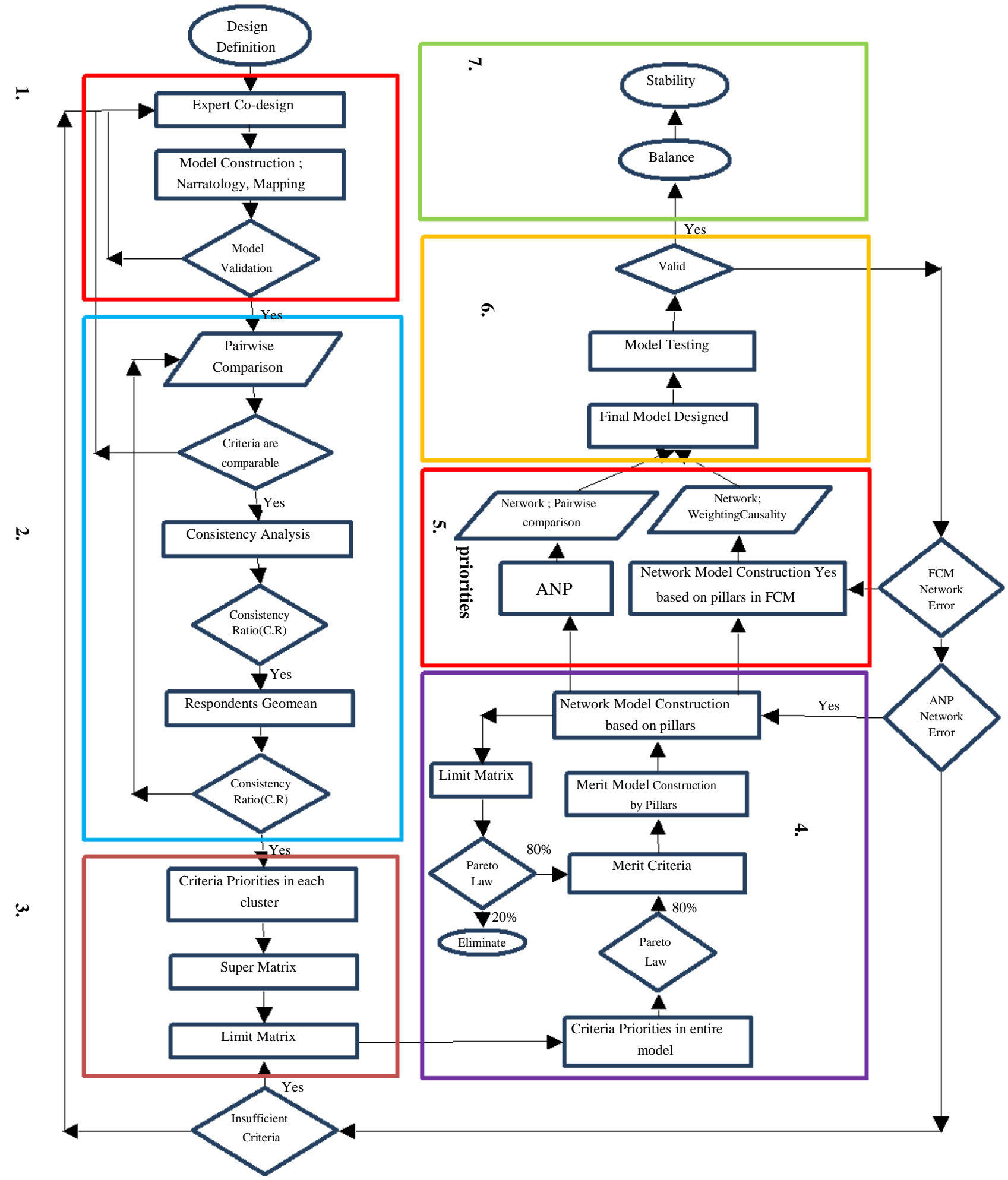

Figure 1: Research Process

International Symposium of

the Analytic Hierarchy

Process
Washington, D. C.

June 29 - July 2, 2014 
- the fostering of a high level of trust amongst all the members of the design team, operations experts as well as researchers. A first step towards this aim was done by the mental mapping, constructed on the basis of experts experience, perceptions and understandings of their system. This was a multi-dimensional, multi-level and multicriteria tool for recognizing the plurality of expert' s perceptions and values as well as for filtering, organizing and storing heterogeneous data. This method also allowed the use of analogies in the cognitive process which prove to be a powerful mean to represent the process under a new and large focus and to generate predictions on what should happen if some elements were modified (Collins and Gentner, 1987). A frequently used metaphor was the one of electric grids so that the system under study could be analysed in terms of electric impulses arousing activity from cluster to cluster thus importing relational structures from one domain to another.

- the identification of design oriented useful data. The collection of data has been done on a situated basis, that is data was carefully chosen not to cover all the system' s reality but most importantly, the just enough data provided it to be coherent and scalable. Two citations inspired the data selection:

Before we can assign numbers to our observations, we must understand the process by which we obtained them in the first place. Gerald Weinberg, 1993

Now, things do not, in general, run around with their measures stamped on them like the capacity of a freight-car : it requires a certain amount of investigation to discover what their measures are.

Norbert Wiener, 1921

- the identification of suitable criteria for the application of the ANP methodology: these criteria were identified by experts through the linking of the maps (macro level information) and the data (micro level information), the data being envisaged as possible captors of the system' s grid.

- the elaboration of scenarios issued from ANP analysis in order to equip the team with the information and knowledge necessary to the conceptualization of a new system, that is, mainly, through the establishment of new connections between the grid clusters and criteria. These new connections are aiming at the operating model equilibrium, thus making a distinction between organization and structure. The organization determines the limit not to be overstepped for the system keeping its identity (Maturana \&Varela, 1972).

The cross-disciplinary research design, methodology and interaction modalities are familiar in the academic literature on simplexity (Berthoz, 2009) which proposes not to master nor reduce complexity but to cope with complexity, leaving behind the debate between simple and complicated. Simplexity as a concept can be considered as a heir of Newton's statement about Nature, in turn closely related to the principle of parsimony put forward by Father William of Ockham in the 14th century. Ockham is the father of the efficient reasoning and of the idea that one should not multiply entities beyond necessity 
has been attributed to him under the expression of Occam' s razor. Father Ockham was far from thinking that seven centuries later, that is today, adding more and more entities, products, information systems, rules, guidelines, etc $\cdots$ would become the core of business and that managers and leaders will be disarmed by not knowing how to define when a given situation is beyond necessity or not yet.

In the complicated environment raised up along at least fifty years, operational efficiency seems to be supported by multiple level procedures and rules, more and more regulatory mechanisms and long top down delegation cascades. This has lessen the innovative capacity, the long term view and quality of professional relationships among operators but also among the hierarchy.

Coping with complex environments and organisations is essentially a matter of relations. This means the ways people put themselves together to act, how these ways are designed by the internal explicit and tacit rules and guidelines, which attitudes are expected, what can be said to whom and when. It is amazing to observe how much sapience is embedded in operators, how much experience is accumulated in experts' minds and how little of this is put at work in decision making processes not to say organizational design initiatives.

To understand complexity we can learn from disciplines like physics or biology when they define, for ex, the operational efficiency of a membrane, the elasticity of a movement or the inter cellular transport of micro fluids. Some attempts to understand complexity in other sectors under this light have explored, for example, the models which may describe financial complexity as if it was similar to the movement of molecules in a gas or the dynamics of stars in a galaxy. Banks now recognise that they have real practical use - for instance, in pricing financial deals - by modelling the markets as 'almost random' processes and then calculating probabilities of future market movements. Operational efficiency can also take advantage of this kind of research, especially to imagine new, simple (simplex) ways of working.

We can hypothesize that mapping techniques allowing the visualization of the information as well as its context and dynamics can be put to work to the benefit of simplexity.

The main stakes related to the way the experts identified criteria, their reaction towards pair-wise comparisons, consistencies and inconsistencies as well as how aggregations took place are presented here. To start with, we find it necessary to ask the experts in the three different groups to describe their organisational system "from the governance to the tools' in their own words without referring only to the official documents such as procedures, process charts, flow charts or strategy presentations. All these documents have no links one with another, they describe different realities inside the company and are, so to say, not supposed to communicate. There was therefore a first issue that was to tell the whole story and to draw the global map of their world putting together information and knowledge about what we defined as being the journey 'from the governance to the tools' across 'the 5 continents' (or clusters : governance, strategy, operations, procedures and tools). We asked them to identify key components of these five clusters. We call these first maps, draw with coloured pencils, structural maps as they are mostly composed of units, departments, committees, rules, etc and say more 
about what things are than how things actually get done. This first step made it clear (for them and for us) that there was no whole spectrum data easily available that we could use to build upon, but also that we will have to use some measurable and many sloppy factors to describe the system.

ANP appeared then to be the most adapted method because of its ability to associate heterogeneous factors and its friendliness to rough data. We then have to introduce the method to the experts especially the idea that we will deal with their subjectivity rather than try to avoid it and that not only the assessment of the current operating model but also the design of alternative ones will be based on their judgments through the pair-wise comparison of the relative weight of the criteria. Prior to using ANP to assess the balance of the operating models we had to redesign the map in order to go from the structural map of clusters to a functional map of clusters and criteria. This change of focus in the way of talking about and representing their system was a turning point in the knowledge experts have about their system. The functional map is not so much about what doesn' t work as improving the model is not our goal. It builds on the knowledge of how things work to describe what is produced (work, knowledge, learning, errors, opportunities, behaviors, values) throughout the daily life of the organization. Thanks to the lay out provided by the map (representing the clusters of criteria and despite two important difficulties (the prospective nature of the goal, the relatively abstract or polysemic nature of the criteria), experts were able to engage into the pair-wise comparisons.

When inconsistencies appeared and differences in experts' judgments were obtained, they were used as opportunities to elicit the reasoning behind weighting and to discuss and clarify the meaning and relevance of the criteria. Even more interesting were the discussions about the aggregate weighting getting one step ahead the goal of getting away from a limited view on the performance of operating models defined in terms of costs reduction and moving towards the definition of what a balanced operating model

This process took over a year to get completed, on the basis of a regular four-hour meeting every 3 weeks. That amount time was needed to go through the different phases and the changes they imply in terms of tasks and relationships. Drawing the first structural map (about "what" are the structural elements of the system) across the socalled ' 5 continents' was unusual but less challenging than the one where experts were asked to talk about "how" the system functions. Then subjectivity becomes involved and different postures emerge, from those pretending that the system functions the way it is supposed to, to more critical voices on the difficulties, drawbacks and dysfunctional consequences of past decisions. Spending time criticizing the current operating model was a drift not to be taken for two reasons, at least: firstly, it could make some group members engaged in current organizing process and projects feeling uncomfortable; secondly, it could limit the discussion to complaining and thus prevent a prospective stance to emerge. Introducing ANP and practicing pair-wise comparisons, was the occasion to go one step further, recognizing the legitimacy of subjectivity in the design process. A high level of trust is a necessary condition to ask people to put the subjectivity at work and engage in working differently in terms of tasks and relationships.

Castelfranchi and Falcone (2000) stress the importance of trust, particularly to go beyond a mere quantitative, probabilistic and opaque view of trust supported by Economics and

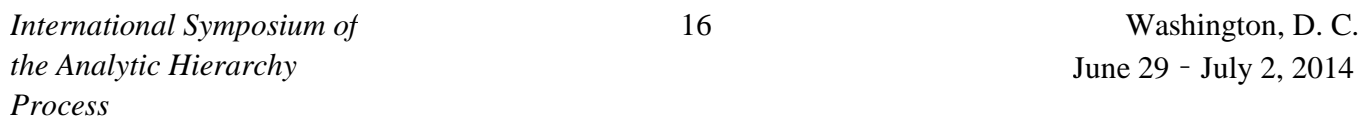


Game Theory. The authors speak in favor of a cognitive view of trust as a complex structure of beliefs and goals encompassing rational and irrational components. The question of trust seems central to the co-designers teams and so at different levels: trust in the team' s judgment as a whole beyond personal expert judgments, trust in the collective eliciting of the gaps between the prescribed and the real which inevitably puts actors at stake, trust in individual and team capacity to imagine a totally new operating model issued from and not just improving or optimizing actual dysfunctions. We thus make the hypothesis that subjectivity is an element of trust within design approaches and ANP has proven a useful tool to situate subjectivity and create value from this.

Different disciplines show today a marked revival of interest in-and legitimation ofthe deliverances of subjective reflection, subjects being at once products and agents of history, agents of knowing as much as of action. Subjective techniques and methods of enquiry are thus critical in the study of complex systems functioning

When faced with a new operating model' $\mathrm{s}$ design in a complex intangible services system, it shall be kept in mind that there is no precise method to measure criteria such as power, influence, political effectiveness, cultural gaps.. (Saaty, 2008). The issue of subjective judgments shall be carefully addressed.

For making relative weighting, AHP \& ANP method uses a scale (Saaty, 2008) which is from one to nine as well, where nine shows the significant difference between the two objects and shows the dominance of one object over another, just as numbers represent equal importance between two compared objects. Let' s remind that ANP method' s matrixes and super matrixes are reciprocal and always positive one' $\mathrm{s}$. A capital merit in this study is that ANP outputs give consistent data.

The cross-disciplinary method of the current study, which integrates anthropology, organization sciences and operations research focuses on swarm intelligence and collective wisdom. In this context, researchers can explain more in detail the concept of pair-wise comparison to the respondent by visualization of the model' s map and fundamental scale of AHP and ANP approach. We believe this approach makes bilateral benefit for the researchers and for the organization' s experts as respondents and codesigners of the new model thus learning about the system and deeply focusing about what is happening and might happen in the future.

As presented in Figure 1, testing the Consistency Ratio (C.R) of the matrix is a key step of the study. Owing to unavoidable inconsistency between judgments and the need for the matching of priorities by the invariance, it is essential to obtain the priorities in the form of eigenvectors of the matrix of the paired comparisons. The consistency ratio is calculated and examined by the researchers and experts to guide their design process as the objects are being compared. Figure 2 presents the geomean calculated for a part of the model 


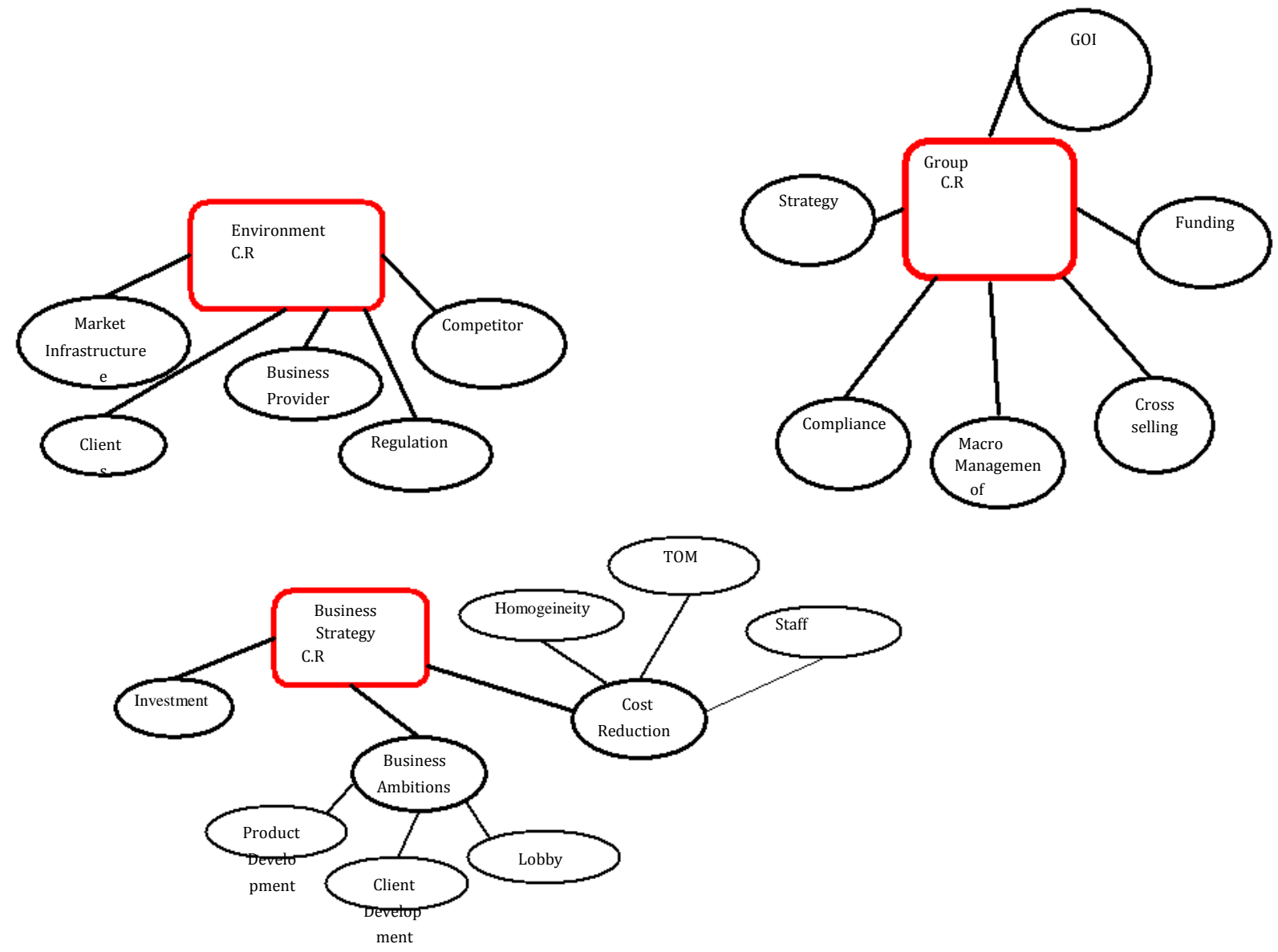

Table 1: A portion of the model's geomean

To analyze the priorities of the criteria and the C.R of the respondents the current study used the latest version 2.2.6 of the Super Decision software.

To quantify the collective wisdom of the experts the geometric mean of them has been computed by the Microsoft Office Excel. The results of the geometric means are used to create "Master" super decision model. The C.R for the geometric mean was computed and followed the rule : C.R must be less than 0.1 .

Table 1 presents a portion of the results of merit and cluster matrix. The same process is undertaken for the whole model. If we take the "environment" cluster example, the two main pillars would be regulation and clients.

\begin{tabular}{|l|l|l|l|}
\hline Cluster & Criteria & Limit & Normalized \\
\hline \hline & 1.1 & 0,018219 & 0,1856 \\
& 1.2 & 0,031599 & 0,3219 \\
& & 0,007968 & 0,08117 \\
& 1.4 & 0,033509 & 0,34135 \\
& 1.5 & 0,00687 & 0,06998 \\
\hline \hline
\end{tabular}

Figure 2: Priorities of the criteria

International Symposium of the Analytic Hierarchy

Process 


\section{6) Limitations}

At this stage of the project, the main limitations can be stated as follows:

--- the heterogeneous kind of data gathered within the system under study composed by data issued from clusters as different as governance, strategy and operations.

--- the lack of literature about the concept of balanced system applied to organizations looked at under a living system/ecosystem metaphor. As usual with metaphors the main limitation is the over parametrization of the model (with many free parameters one can fit any desired behaviour) In the present approach we are not fitting a linear model behaviour but rather estimating the parameters of an "independent experiment" and then comparing qualitative features of different possible behaviours as possible functions of the determined parameters

--- the lack of appropriate benchmark as a stable model designed to compare the existing one. Classical "decision making" oriented studies will use known ratios as, for example, Carbon/Nitrogen one in the soil to estimate deviations and assess judgements. In the case of the study presented here, the pertinent ratios have to be discovered.

--- The relatively abstract or polysemic criteria used in defining the "how" an organization works and the prospective nature of the goal

We feel these limitations are originated in the fact of using a decision making recognized method within a different context, that is, the design of a new system. This question challenges key issues like data gathering, experts participation or consistency and sharpens creativity.

\section{7) Conclusion}

Combining the use of ANP with methods and approaches from organization and anthropological sciences has confirmed our conviction of the ANP method crossdisciplinary friendliness. ANP approach has helped experts to express their views of the system in a multi level way, adapted to the broad operating model study' s spectrum "from the governance to the tools" . It has also allowed to engage an in depth discussion about the role played by subjectivity and intuition in design processes.

ANP provides the substitute of experiments in the determinaion of the model and will again assess relevance structure of the FCM to come. Given the trivalent dynamic characteristics of FCM matrixes, these will provide an aproximation to the posible equilibria and how they control the dynamics of the system.

The next step will be to provide a proof of concept of the possibility of modelling an organization's complex behavior using the living system metaphor with differential equations. We believe this issue is at the centre of the modelling of any socialorganizational process

We end by remarking that only a number of studies in different situations which prove predictive will validate the present approach. 


\section{8) Key References}

Aguilar, J (2005). A survey about Fuzzy Cognitive Maps Papers. International Journal Of computational Cognition, vol. 3, $\mathrm{N}^{\circ}$ 2, 27- 33

Ashkenas, R. (2012) It's Time to Rethink Continuous Improvement http://blogs.hbr.org/2012/05/its-time-to-rethink-continuous/

Axelrod, R. (1976). Structure of Decision. The Cognitive Maps of Political Elites, Princeton University Press, Princeton, NJ

Bellomo, N. Ed (2008). Modeling Complex Living Systems: A Kinetic Theory and Stochastic Game Approach. Modeling and Simulation in Science, Engineering and Technology. Birkhäuser Publications

Berthoz, A (2009). La Simplexité. Odile Jacob.

Cardoen B. et al (2010). Operating room planning and scheduling: A literature review European Journal of Operational Research, vol 201, issue 3, 921-932

Castelfranchi, C \& Falcone, R (2000).Trust Is Much More than Subjective Probability: Mental Components and Sources of Trust. Proceedings of the 33rd Hawaii International Conference on System Sciences, 1-10.

Collins, A., and D. Gentner. (1987). How people construct mental models. D. Holland and N. Quinn, editors. Cultural models in language and thought, 243-268. Cambridge University Press.

Collinson S. (2012) True cost of complexity in the banking sector. Simplicity Partnership.

Da Silva, A.S et al (2009) ANP and ratings model applied to supplier selection problem. Proceedings on the intl symposium on the AHP, 1-14.

Eaton T. (2011). A cultural analysis of information technology offshore outsourcing : an exercice in multi sited ethnography of virtual work. Wayne State university Dissertations, paper 370.

Jones, $\mathrm{N}$ et al (2011), Mental Models: An Interdisciplinary Synthesis of Theory and Methods, Ecology and Society 16 issue1, article 46.

Korff, R. (2003). Local Enclosures of Globalization. The Power of Locality. Dialectical Anthropology 27(1),1-18.

Kosko, B (1986). Fuzzy Cognitive Maps, International Journal of Man-Machine Studies, 24, 65-75 
Martinez-Moyano, I et al (2014). Drift and Adjustment in Organizational Rule

Compliance: Explaining the Regulatory Pendulum. Financial Markets, Organization Science, 2014, 25, 2, 321

Maturana, H. \& F. Varela. (1980) Autopoiesis and Cognition. Boston studies in the philosophy of sciences. D. Reidel Publications

Murray, JD (2008). Mathematical Biology. Springer Verlag.

O'Neil, P. D. and Krane, D. (2012), Policy and Organizational Change in the Federal Aviation Administration: The Ontogenesis of a High-Reliability Organization. Public Administration Review, 72: 98-111

Padillo J M \& M Diaby (1999). A multiple-criteria decision methodology for the makeor-buy problem. International Journal of Production Research, Volume: 37 Issue: 14, 3203-3229

Santiago Medina Hurtado. (2010). Modeling of Operative Risk Using Fuzzy Expert Systems in Michael Glykas (Ed.). Fuzzy Cognitive Maps Advances in Theory, Methodologies, Tools and Applications, Springer.

Saaty, T.L., (1999). Fundamentals of the analytic network process. ISAHP 1999, Kobe, Japan

Saaty, T.L. (2005). Theory and applications of the analytic network process: decision making with benefits, opportunities, costs, and risks. Pittsburgh, PA: RWS Publications.

Saaty, T. L. (2008). The analytic hierarchy and analytic network measurement processes: Applications to decisions under Risk. European journal of pure and applied mathematics.

Staats, B. R., \& David M. Upton. (2007) Lean Principles, Learning, and Software Production: Evidence from Indian Software Services Harvard Business School Working Paper. No. 08-001.

Stylos, C \& Groumpos, P. (1999). Mathematical formulation of fuzzy cognitive maps. Proceedings of the 7th Mediterranean Conference on Control and Automation (MED99) Haifa, Israel, 2251- 2261

Thomas WI \& F Znaniecki (1918-1920) The Polish peasant in Europe and America. Monograph of an immigrant group. Univ of Chicago Press.

Weick KE (1987). Organizational culture as a source of high reliability. California Management Review, 29: 112-127. 20.

Weick KE (2011). Organizing for transient reliability: the production of dynamic nonevents. Journal of Contingencies and Crisis Management, 19: 21-27 
Weick KE, Sutcliffe KM, Obstfeld D (1999). Organizing for high reliability: processes of collective mindfulness. Research in Organizational Behavior. Volume 21. Edited by Sutton RI, Staw BM. Greenwich, CT: JAI Press, 81-124

Weinberg, G. (1993). Quality Software Management. Dorset House.

Wiener, N. (1921) A New Theory of Measurement: A Study in the Logic of Mathematics Proceedings of the London Mathematical Society, Volume 2-19 Issue 1 